\title{
Guarding the perimeter: protection of the mucosa by tissue-resident memory T cells
}

\author{
LS Cauley $^{1}$ and L Lefrançois ${ }^{1}$
}

Mucosal tissues are continually bombarded with infectious agents seeking to gain entry into the body. The absence of a tough physical exterior layer surrounding these tissues creates a unique challenge for the immune system, which manages to provide broad protection against a plethora of different organisms with the aid of special adaptations that augment immunity at these vulnerable sites. For example, specialized populations of memory $\mathrm{T}$ lymphocytes reside at initial sites of pathogen entry into the body, where they provide an important protective barrier. Similar anatomicallyconfined populations of pathogen-specific CD8 T cells can be found near the outer margins of the body following recovery from a variety of local infections, where they share very similar phenotypic characteristics. How these tissueresident T cells are retained in a single anatomic location where they can promote immunity is beginning to be defined. Here, we will review current knowledge of the mechanisms that help establish and maintain these regional lymphocytes in the mucosal tissues and discuss relevant data that enhance our understanding of the contribution of these lymphocyte populations to protective immunity against infectious diseases.

\section{INTRODUCTION}

Respiratory infections claim the lives of $\sim 4$ million people worldwide each year. This number increases dramatically during sudden outbreaks with highly pathogenic respiratory viruses, including new strains of influenza virus. ${ }^{1,2}$ Enteric infections and sexually transmitted diseases are also responsible for high casualty rates, which can be particularly devastating in under-developed countries. ${ }^{3}$ A thin epithelial layer makes mucosal surfaces particularly vulnerable to pathogens, which often take advantage of specific host receptors to gain entry into the body. ${ }^{4,5}$ The immune system uses a variety of means to repel these infections, including nonspecific secretions that contain mucins and defensins. ${ }^{6,7}$ Over time antigen-specific lymphocytes develop and help reduce tissue damage and hematogenous dispersal of the pathogen to other regions of the body.

Adaptive immune responses to pathogens that have not been encountered previously take several days to develop; hence, innate immune cells must provide the first line of defense. ${ }^{8}$ Early warnings of the invasion begin as receptors that recognize common microbial products (called pathogen-associated molecular patterns) elicit cytokine responses in the local tissues and arm cells of the innate immune system for combat. ${ }^{9-11}$ Antigen-specific lymphocytes are recruited into the response once professional antigen-presenting cells, primarily dendritic cells (DCs), reach, or are activated in, the local lymphoid tissues. ${ }^{12,13}$ Although some microbial products access the local lymphoid tissues within minutes or hours after inoculation, a substantial delay follows because several rounds of proliferation are required for naive $\mathrm{T}$ cells to reach therapeutic numbers that can potentially change the course of the infection. ${ }^{14}$ This delay in the development of the adaptive immune response may allow some pathogens to spread to other tissues where timely eradication may not be possible.

People and animals that survive an initial infection often gain a survival advantage during reinfection with the same pathogen because they maintain populations of antigen-specific lymphocytes that can mount faster more effective responses to the same microbe. ${ }^{15}$ These long-lived lymphocytes are collectively known as memory cells, but can be very heterogeneous in terms of phenotype, functional properties, and tissue distribution. ${ }^{16-18}$ During recent years, it has become increasingly clear that different subsets of antigen-specific $\mathrm{T}$ cells make varying contributions to protective immunity. Although CD4 T cells

${ }^{1}$ Department of Immunology, Center for Integrated Immunology and Vaccine Research, UCONN Health Center, Farmington, Conneticut, USA. Correspondence: L Lefrançois (llefranc@neuron.uchc.edu) 
are clearly involved in protection against some mucosal infections, ${ }^{19-21}$ to date most research has been focused on understanding the contributions of circulating and tissueresident CD8 memory T cells. These studies show that some of the properties that are important for immunity include the anatomical location of the CD8 T cells at the time of infection and their ability to mediate specific effector functions with minimal delay. ${ }^{18}$ Building on observations from earlier studies, several investigators have recently focused on subsets of memory CD8 T cells, which take up long-term residence in nonlymphoid tissues where they remain phenotypically and functionally distinct from their circulating counterparts. Recent data suggest that these $\mathrm{T}$ cells are able to make important contributions to immunity against localized infections in peripheral tissues such as the lung, intestine, and skin. ${ }^{18,22} \mathrm{We}$ will discuss our current understanding of the mechanisms that support the development, maintenance, and function of these local pathogen-specific $\mathrm{T}$ cells in the mucosal tissues of the gastrointestinal (GI) and respiratory tracts.

\section{INDUCTIVE SITES FOR IMMUNE RESPONSES IN THE MUCOSAE}

Evidence suggests that most tissue-resident cytotoxic $\mathrm{T}$ lymphocytes (CTLs) in the intestinal and pulmonary mucosae begin their development at specific inductive sites in the local lymphoid tissues and subsequently move into the mucosae during robust inflammation. ${ }^{23-25}$ Migration studies indicate that as inflammation resolves, the tissue-resident CTLs become less mobile and lose the ability to re-enter the circulation. Many properties that enable $\mathrm{T}$ cells to traffic to specific locations in the body are programmed during the early stages of the infection, through signals that are delivered by antigen-presenting cells and local environmental factors.

The mucosal tissues of the GI and respiratory tracts have similar functions, which involve exchange of diffusible molecules, but some notable differences in their structural organization influence the way that local immune responses develop. As in other tissues, adaptive immune responses in the mucosae begin in organized lymphoid tissues, which serve as collection points for microbial products and immune cells. The mesenteric lymph nodes (MLNs) and the Peyer's patches (PPs) fulfill the role of inductive sites for immune responses against pathogens that enter the body via the intestine. ${ }^{26,27}$ Multiple PPs are distributed along the length of the small bowel, where they can receive microbial products from the lumen of the intestine. Isolated lymphoid follicles, which develop after birth, can fulfill similar functions in the small intestine and colon. ${ }^{28,29}$ In addition, a string of encapsulated MLNs is connected to the afferent lymphatics of the PPs and lamina propria (LP), where migratory cells (DC and lymphocytes) collect after they leave the intestinal tissues (Figure 1). ${ }^{13,30}$

Adaptive immune responses against pathogens that enter the body from the respiratory tract are also initiated in multiple locations. The regional lymphoid tissues include two collections of encapsulated LNs, which serve the upper (nasal passages) and lower (lungs) regions of the respiratory tract,

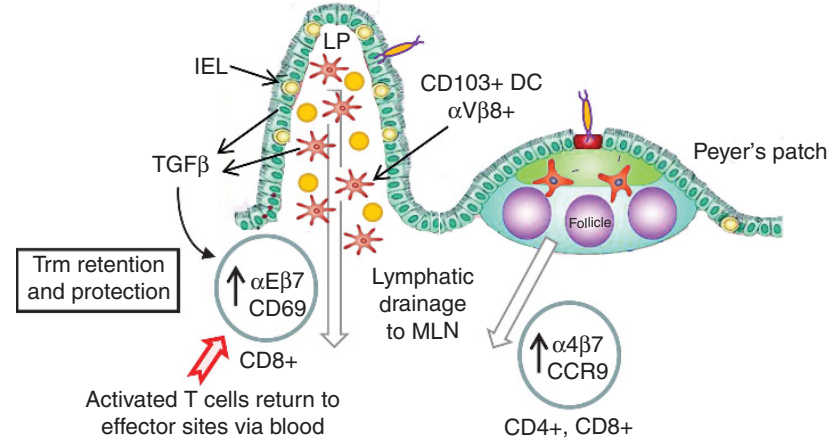

Figure 1 Anatomic and cellular features of the intestinal mucosal immune system. Priming by specialized dendritic cells (DCs) in the immune-inductive sites of the intestine, the Peyer's patch, and the mesenteric lymph node (MLN) results in the induction of intestine-specific homing molecules, $\alpha 4 \beta 7$ and CCR9, which allows activated T cells to enter the effector sites, the lamina propria (LP) and the epithelium. Local effects of tumor growth factor $\beta$ (TGF $\beta$ ) induce $\alpha \mathrm{E} \beta 7$ expression (CD103 $=\alpha \mathrm{E}$ ), and CD69 is induced by unknown factors. CD103 promotes T-cell retention in the epithelium, and CD69 may have similar effects by downmodulating the activity of sphingosine-1-phosphate receptor 1 (S1P1). Retention of regional or resident memory T cells (Trms) enhances protection to reinfection. IEL, intraepithelial lymphocyte.

respectively. During infections microbial products are acquired from the airways and nasal passages by a tight network of respiratory DCs, which reside in the epithelium and LP of the lungs. ${ }^{31-33}$ Migratory DCs that obtain antigens in the upper respiratory tract migrate to the cervical LNs, whereas the mediastinal (or hilar) LNs receive DCs that acquire antigens in the lungs. ${ }^{34,35}$ After birth, additional patches of nasopharynxassociated lymphoid tissue (i.e., the equivalent of adenoids or tonsils in human) develop in the nasal passages of the murine and human respiratory tract, which are a major inductive site for B-cell responses. ${ }^{36-38}$ The mucosal-associated lymphoid tissues lack afferent lymphatics; thus, antigens must be transported across the epithelium by local DCs, or specialized $\mathrm{M}$ (microfold) cells, which can be found on the apical dome of the intestinal PPs and nasal epithelium. ${ }^{39,40}$ Children sometimes develop additional patches of ectopic or inducible lymphoid tissue in the lungs, which develop at the bifurcations of the bronchi during inflammation (inducible bronchus-associated lymphoid tissue), but are generally not found in adults. ${ }^{41}$

Lymphocyte activation can take place in any individual PP, MLN, or lung-draining LN and lead to specific humoral and cellular immune responses in the infected tissue. The specialized cells that participate in these immune responses include multiple subsets of migratory DCs. ${ }^{13,31,42}$ Naive CD8 T cells can be primed directly in the PPs or in the MLNs, or in the lung-draining LNs by DCs that migrate from the PPs or LP, ${ }^{13,43}$ or the lung. ${ }^{31,44}$ DCs that express the $\alpha E \beta 7$ integrin (CD103) have an important role in the response by carrying microbial products to the draining LNs, ${ }^{33,45}$ although some CD103-DCs can also serve this function ${ }^{33,43}$ (Figure 1). Immune activation by specialized DCs can lead to tolerance or immunity depending on the context of antigen exposure. ${ }^{45,46}$

An extensive body of literature shows that local DCs in the PP and MLN imprint newly activated antigen-specific CD4 and 
CD8 T cells for preferential migration to the small bowel. ${ }^{47-49}$ The responding T cells acquire homing receptors, CCR9 and $\alpha 4 \beta 7$ integrin, which are essential for entry into the intestinal LP and are induced by factors produced by DCs and intestinal epithelial cells, primarily retinoic acid and transforming growth factor- $\beta$ (TGF $\beta$ ) ${ }^{50-54}$ The $\alpha 4 \beta 7$ integrin facilitates lymphocyte access into the gut mucosa through interactions with the mucosal addressin, MAdCAM-1, that is expressed in the postcapillary venules. ${ }^{55,56}$ When the $\alpha 4 \beta 7+\mathrm{CD} 8 \mathrm{~T}$ cells arrive in the intestinal mucosa, the $\beta 7$ chain of the integrin heterodimer acquires a new partner (CD103, the $\alpha \mathrm{E}$ chain) whose expression is induced by local exposure to CCL $25^{57}$ and TGF- $\beta .^{53,58}$ In the LP, $\sim 50 \%$ of the CD8 T cells express CD103, whereas in the intraepithelial lymphocyte (IEL) compartment, nearly all of the cells are $\mathrm{CD} 103+.^{59,60}$ A population of activated $\mathrm{CD} 4 \mathrm{~T}$ cells also acquire gut-homing properties in the MLNs by upregulating $\alpha 4 \beta 7$ and CCR9. ${ }^{61}$ In addition, a subset of CD4 T cells upregulate CD103 upon entry into the epithelium, but not to the same extent as CD8 T cells. ${ }^{62}$

Although CD103 + DCs participate in immune responses in the respiratory tract, ${ }^{33,63}$ no clear evidence that antigen-specific CD8 $\mathrm{T}$ cells can be imprinted for preferential migration to the lungs has yet been reported. However, a recent study found that when virus-specific CD4 T cells isolated from the lungs after influenza virus infection are transferred to uninfected mice, they preferentially localize to the lungs, whereas spleen-derived cells disperse to multiple tissues, including the spleen. ${ }^{64}$ In light of the similarities between the mucosal tissues of the GI and respiratory tracts, it seems likely that imprinting functions for pulmonary DCs will be identified in future studies.

\section{CIRCULATING LYMPHOCYTES ARE LARGELY EXCLUDED FROM THE MUCOSAE UNDER HOMEOSTATIC CONDITIONS}

The bloodstream is an important access route for migrating $\mathrm{T}$ cells to reach different tissues. Parabiosis has been effectively used to distinguish between cells that freely traffic around the body from cells that take up long-term residence in specific tissues. ${ }^{65-68}$ Within a few days after parabiosis surgery, bloodborne $\mathrm{T}$ cells begin to travel freely between the conjoined animals and congenic markers can be used to measure the rate and extent of intermingling between donor and recipient cells in different organs. ${ }^{68}$ Although mice cannot be joined during robust inflammatory responses, the surviving memory $\mathrm{T}$ cells can be analyzed during the recovery phase of an infection. Such experiments have shown that memory $\mathrm{T}$-cell migration to certain tissues is highly regulated. For example, the migration patterns of polyclonal $\mathrm{T}$ cells as well as antigen-specific memory CD8 $\mathrm{T}$ cells in mice that had been infected with vesicular stomatitis virus or Listeria show that entry into the LP and epithelium of the intestine is highly restricted under homeostatic conditions. ${ }^{68,69}$ Experiments with mice that had recovered from systemic viral or bacterial infections before they were joined to uninfected partner animals showed that only small numbers of circulating memory CD8 T cells enter the LP of the intestine and are almost completely excluded from the epithelial layer. ${ }^{68}$ Nevertheless, the limited migration that occurs is $\alpha 4 \beta 7$-dependent. ${ }^{68}$ Although an ongoing, albeit minor, contribution of new cells may enter the intestinal pool from the blood during the memory phase of the infection, data from our laboratory and others support the concept that the pool of memory $\mathrm{T}$ cells that colonizes intestinal effector sites under steady-state conditions includes tissue-resident, selfrenewing cells that undergo very limited replenishment from the circulating lymphocyte pool. ${ }^{60,68,70,71}$

Parabiosis has also been used to examine the migration properties of blood-borne lymphocytes to the lungs. ${ }^{68,72,73}$ Blood enters the lungs from two separate circulatory systems. The conducting airways have thick walls, which are nourished with arterial blood, whereas the remaining parenchymal tissue is largely comprised of thin-walled alveoli, which are profusely supplied with venous blood that is delivered to the lungs for gaseous exchange. When mice that have recovered from a systemic viral infection are joined to uninfected animals, bloodborne memory CD8 $\mathrm{T}$ cells freely circulate through the parenchymal tissues of the lungs, but do not enter the lumen of airways under homeostatic conditions. ${ }^{68,72}$ However, recent studies have shown that substantial numbers of lung-resident CD4 and CD8 T cells may, in fact, be trapped in the capillaries. ${ }^{64,74}$ When anti-CD8 antibodies are injected into the bloodstream just a few minutes before the cells are isolated, $\sim 95 \%$ of memory CD8 T cells in systemically infected mice are labeled with the blood-borne antibodies, indicating that most of the cells are intravascular. ${ }^{74}$ Similar results are obtained regardless of whether or not the mice have been perfused of blood. These results suggest that perfusion does not remove a large fraction of $\mathrm{T}$ cells from the small blood vessels of the lungs and support the idea that only small numbers of circulating memory $\mathrm{T}$ cells extravasate into the parenchyma in the absence of a robust inflammatory response. Indeed, experiments with pertussis toxin show that migration of transferred memory $\mathrm{T}$ cells into the lung is chemokine receptor-dependent. ${ }^{74}$ Thus, the interpretations drawn from results of parabiosis studies, and from studies using simple digestion techniques, regarding the locations of $\mathrm{CD} 8 \mathrm{~T}$ cells in the lungs, need to be reconsidered in light of the new findings. Moreover, these findings effectively reconcile the results showing limited potential for pathogen-specific memory $\mathrm{T}$ cells to reach the outer mucosal surfaces of the intestines and lungs.

The presence of infection or inflammation in the lungs can affect the migration of effector and memory $\mathrm{T}$ cells. Earlier studies show that circulating memory $T$ cells can be drawn into the lung airways during systemic infections, or by treating the lungs with Toll-like receptor ligands or other agents to induce local inflammation. ${ }^{23,24,73}$ These approaches show that very few CD8 $\mathrm{T}$ cells persist or maintain an activated phenotype in the lumen of the airways in the absence of their cognate antigen. When parabiotic mice are infected with influenza virus before they are joined to uninfected partners, very few migrating CD8 $\mathrm{T}$ cells enter the airways of the uninfected animals. ${ }^{73}$ However, when both mice are previously infected with the same strain of influenza virus, a subset of memory CD8 T cells from each partner enters the lungs of the partner parabiont, is distinct 
from the resident CD8 T cells and does not become activated. ${ }^{73}$ The same experiments show that donor and recipient virusspecific CTLs also equilibrate at very different rates in the draining vs. remote $\mathrm{LNs} .{ }^{73}$ In this case, the prolonged retention of virus-specific CTLs in the lung-draining LNs corresponds with the prolonged presence of residual viral peptides and the numbers of CD69 + PD-1 + CTL. ${ }^{73,75,76}$ These results imply that close interactions with antigen-bearing DCs, and/or an ongoing response to residual antigen-driven inflammation, ${ }^{77-79}$ promote retention of antigen-specific CD8 $\mathrm{T}$ cells in reactive LNs. It is likely that the local inflammatory response is augmented by the presence of virus-derived genetic material, which is detectable in the lungs ${ }^{76}$ and mediastinal LN more than a month after infection (M. Wu and L.S. Cauley, personal communication). Genetic material and viral protein also persist in the LNs after systemic infection with $\mathrm{VSV},{ }^{80-82}$ suggesting that antigen persistence after RNA virus infections may be more common than previously appreciated. How these viral products influence the activation state and migratory properties of the local virus-specific $\mathrm{T}$ cells remains to be determined.

\section{REGIONAL LYMPHOCYTE POPULATIONS COLONIZE THE INTESTINAL AND RESPIRATORY MUCOSAE}

Cell populations with the phenotypic characteristics of regional or resident memory $\mathrm{T}$ cells (Trms) have been detected in a variety of peripheral tissues, including the intestine, lung, skin, kidneys, pancreas, and brain. ${ }^{68,73,83-90}$ The hallmarks of regional or tissue-resident memory CD8 T cells (Trms) include the absence of CCR7, elevated CD103 expression that is regulated by TGF $\beta,{ }^{58,91}$ and often increased CD69 expression. The tissue-restricted distribution of cells with this phenotype suggests that tissue-resident memory $\mathrm{CD} 8 \mathrm{~T}$ cells are influenced by, or formed in response to, factors present in the local tissues.

An extensive population of Trms resides in the GI tract, most prominently among the CD $8 \alpha \beta$ IELs located between the epithelial cells that cover the basement membrane of the villi in the small bowel (Figure 1). The intestinal LP also harbors a significant population of $\mathrm{CD} 8 \alpha \beta$ Trms. Nevertheless, a clear distinction between the epithelial and LP compartments is exemplified by the distinct cell populations at each location, as the IELs include subsets of T-cell receptor (TCR) $\gamma \delta \mathrm{T}$ cells as well as many $\mathrm{TCR} \alpha \beta \mathrm{T}$ cells that express $\mathrm{CD} 8 \alpha \alpha$, which are not found in the LP. ${ }^{92,93}$ Smaller numbers of CD4 T cells, and some unusual CD $4+8 \alpha \alpha+$ cells, also reside in the epithelium. ${ }^{93}$ The underlying LP contains a more complex mix of T cells, antigenpresenting cells, plasma cells, and natural killer cells among others. The T cells in the LP also include smaller percentages of $\mathrm{TCR} \gamma \delta$ cells that express substantially different V-regions than those in the IEL compartment. As the TCR repertoires of TCR $\alpha \beta$ cells in the LP vs. the IEL can also be distinct, ${ }^{94,95}$ it is likely that little intermixing occurs between the IEL and LP populations under steady-state conditions. Whether the Trms that reside in the LP and IEL compartments are equivalent cell populations or distinct subsets is not yet known. In any case, large numbers of effector $\mathrm{T}$ cells enter all parts of the intestine during local and systemic infections, when some effector/ memory T cells traverse the LP to reach the epithelial layer. ${ }^{25}$ Following the contraction of the effector response, a subset of pathogen-specific CD8 $\mathrm{T}$ cells in the LP, and nearly all of the cells in the IEL compartment, acquire CD103 and CD69. ${ }^{60,68,96}$ The induction of CD69 and CD103 is antigen-independent in the intestine, as demonstrated by parabiosis studies where memory CD8 $\mathrm{T}$ cells enter the mucosa of the uninfected partners and upregulate both molecules without antigen stimulation. ${ }^{68}$ Thus, these events are a prime example of local, antigen-independent tissue-specific effects regulating Trm development.

The characteristics of the T-cell response in the respiratory tract follow a similar pattern. During infection, intense inflammation draws large numbers of lymphocytes into the airways and alveoli of the lungs, which include both pathogenspecific and bystander CD4 and CD8 T cells ${ }^{23,24}$ (Figures 2 and 3). The number of $\mathrm{T}$ cells in the lungs decreases as inflammation subsides, but residual populations of pathogenspecific CTLs persist in the airways for several months after some infections. ${ }^{76,97}$ The phenotypic characteristics of the CD8 $\mathrm{T}$ cells that reside in the lungs after a local viral infection are remarkably similar to those found in the GI tract. For example, many virus-specific CTLs upregulate CD69 and CD103 expression and become greatly enriched in the airways over time. $^{91,98-100}$ Unlike IEL in the GI tract, these virus-specific CTLs lose CD11a expression soon after their arrival in the lumen of the airways, ${ }^{25,101,102}$ presumably due to cleavage by proteases present in the lung surfactant. The loss of CD11a expression can be used as an indication of recent migration into the airways, ${ }^{73,102}$ and the data support the conclusion that small

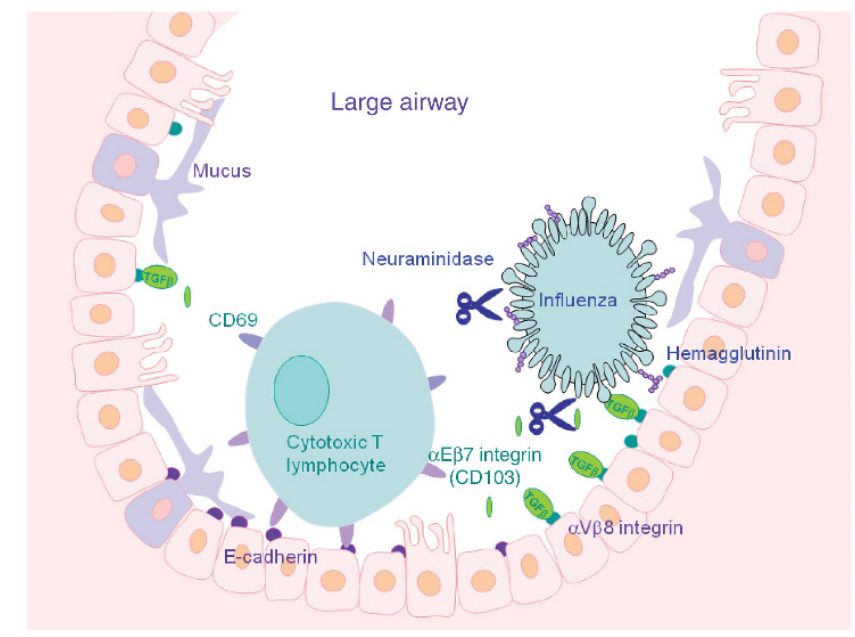

Figure 2 Parameters affecting the CD8 T-cell response to influenza virus infection. Influenza virus infections begin in the large airways and descend to the alveoli. Hemagglutinin binds to sialic acid residues on the surface of the epithelial cells, which become infected and produce new virus. Neuraminidase helps the new virus escape from the host cells by removing host sialic acid. Mucus-producing and ciliated cells slow the infection by expelling particulate matter from the lung airways. Neuraminidase and the $\alpha \mathrm{V} \beta 8$ integrin can activate tumor growth factor- $\beta$ (TGF $\beta$ ), which promotes $\alpha \mathrm{E} \beta 7$ integrin (CD103) expression on local pathogen-specific cytotoxic $\mathrm{T}$ lymphocytes, which promotes retention in the airways through interactions with E-cadherin. 


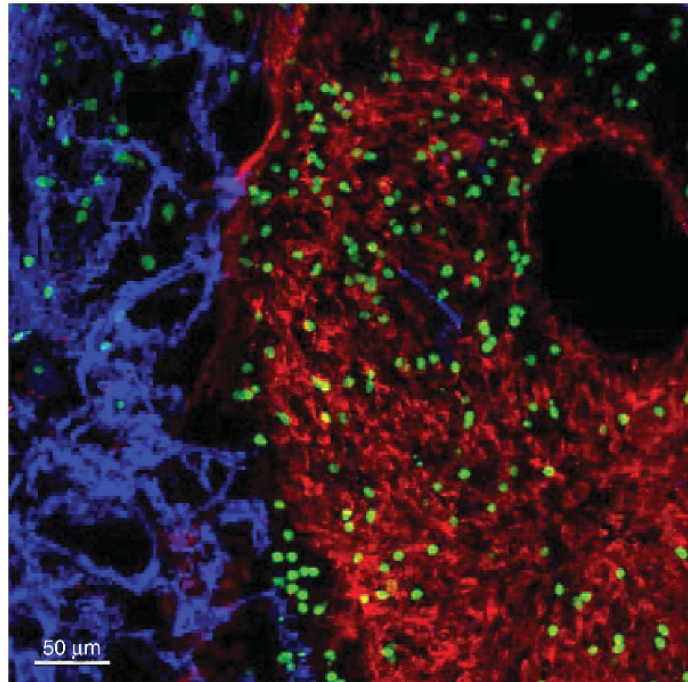

Figure 3 Effector CD8 T-cell localization in the lung after influenza virus infection. C57BL/6 mice received $1 \times 10^{3}$ nucleoprotein (NP)-specific green fluorescent protein (GFP) + F5 T-cell receptor (TCR) transgenic CD8 T cells followed by infection with E61-13-H17 influenza virus. After 10 days, the lung was imaged using confocal microscopy. Epithelial cells were identified by anti-Epcam staining (red) and blood vessels revealed by anti-CD31 staining (blue). GFP + F5 cells are shown in green. Image was acquired at original magnification $\times 20$.

numbers of virus-specific CTLs enter the airways during the recovery phase of the response to respiratory virus infection. In contrast, the virus-specific CTLs in the lung parenchyma maintain CD11a expression at high levels and mostly lack CD69 expression. ${ }^{73,91}$ It is not known how many virus-specific CTLs reside in the dense network of blood vessels that surround the alveoli, but as discussed in the previous section bona fide tissue-resident CD8 T cells may be primarily activated CTLs, or effector memory T cells. ${ }^{64,73,74,91}$

Responses by CD4 T cells in mucosal tissues differ from those of CD8 T cells in several ways. Notably, the numbers of pathogen-specific CD4 $\mathrm{T}$ cells in the lung airways decline much more rapidly after local infections, than do the numbers of virus-specific CTLs. ${ }^{64,97,103}$ A subset of virus-specific memory CD4 T cells maintain stable CD69 expression in the lung parenchyma and airways following respiratory virus infection and some $\mathrm{CD} 4 \mathrm{~T}$ cells in the airways downregulate CD11a. ${ }^{97,102}$ These CD69 + CD4 T cells do not emigrate from the lungs during parabiosis and are not labeled with injected anti-CD4 antibodies, indicating that they are part of the tissue-resident memory population. ${ }^{64}$ Importantly, CD4 $\mathrm{T}$ cells that were isolated from the lungs of influenza virus-infected mice were sufficient to enhance immunity after transfer to naive recipients, without the need for transferred virus-specific memory CD8 T cells. ${ }^{64,104,105}$ Whether CD4 T cells make a direct contribution to viral clearance during natural infection has not been established. Some CD4 T cells in the GI tract also maintain an activated phenotype and a recent study found that sustained CD69 expression was maintained by normal intestinal microflora and interferon $\alpha^{106}$ Taken together, these data indicate that the LP of the gut and the lung parenchyma provide similar microenvironments, which can be colonized by resident populations of memory CD4 and CD8 $\mathrm{T}$ cells following infection.

\section{RETENTION AND EXIT OF PATHOGEN-SPECIFIC T CELLS FROM THE MUCOSAE}

CD69 is a highly glycosylated C-type lectin, which is briefly expressed on CD4 + and CD8 $+\mathrm{T}$ cells soon after TCR stimulation. ${ }^{107,108}$ Transient CD69 expression can also be induced by type I interferon, but disappears upon cytokine withdrawal, at least in vitro. ${ }^{109}$ As discussed above, antigenspecific CD4 and CD8 T cells that maintain stable CD69 expression can be found in the respiratory tract of mice following infection with a variety of viruses ${ }^{91,98-100}$ and similar cells have been identified in human lungs. ${ }^{88}$ CD $69+$ CTLs are continually present in both the LP and IEL compartments of the GI tract of mice ${ }^{110,111}$ and are sometimes found in peripheral tissues, such as the skin, ${ }^{89,112}$ trigeminal ganglia, ${ }^{113,114}$ brain, ${ }^{87}$ and kidneys. ${ }^{83}$ The mechanisms that support sustained CD69 expression have not been completely defined, but antigen stimulation appears to have a role in some infections and some tissues, ${ }^{87,91}$ but not others. ${ }^{68,90}$

Accumulating data indicate that CD69 and CD103 both have a role in populating peripheral tissues with activated CTLs. ${ }^{58,91}$ The natural ligands for CD69 have not been conclusively identified, but are thought to include complex oligosaccharides. ${ }^{115}$ Earlier studies show that overexpression of CD69 slows the rate at which naive $\mathrm{T}$ cells exit the thymus, ${ }^{116,117}$ whereas CD69-deficient T cells exit peripheral LNs more rapidly than their wild-type counterparts after activation with poly-I:C or viral infection. ${ }^{118}$ The lipid sphingosine-1-phosphate (S1P) has an essential role in lymphocyte migration ${ }^{119}$ and evidence of interacions between CD69 and the S1P receptor 1 (S1P1) may provide clues to CD69 function in tissue retention of Trms. ${ }^{118,120}$ The S1P1 receptor is expressed on T cells and is essential for thymocytes to reach the periphery. As naive lymphocytes circulate around the body, S1P1 is also essential for naive lymphocytes to exit the LNs. ${ }^{121} \mathrm{CD} 69$ interacts with the transmembrane region of $\mathrm{S} 1 \mathrm{P} 1,{ }^{120}$ resulting in downmodulation of S1P1 function, suggesting that CD69 upregulation could potentially modulate the ability of $\mathrm{T}$ cells to exit nonlymphoid tissues. For example, induction of CD69 expression as memory or effector $\mathrm{T}$ cells that enter the mucosal tissues might serve to downregulate S1P1 expression and thus reduce responsiveness to $\mathrm{S} 1 \mathrm{P}$, which is maintained at the highest concentrations in the blood and lymph. Indeed, under homeostatic conditions the S1P1 receptor has a role in the differential migration of $\mathrm{T}$-cell subsets into distinct intestinal compartments. ${ }^{62}$

The chemokine receptor CCR7 also has an integral role in mediating T-cell exit into afferent lymphatics from nonlymphoid tissues ${ }^{122-124}$ and is important for DC migration from peripheral tissues to the draining LNs. ${ }^{124,125}$ During influenza virus infection, the exit of effector CD8 $\mathrm{T}$ cells from the lungs is facilitated by the CCR7 ligand, CCL21, which is expressed in lymphatic endothelial cells during infection. ${ }^{126}$ Once CCR7 is downregulated, the rate of T-cell migration from the lung 
tissues to the mediastinal $\mathrm{LN}$ diminishes. In contrast, forced expression of CCR7 results in more rapid exit of effector T cells from the lung, ${ }^{122}$ whereas in another model CCR7 deficiency prevents tissue-resident lymphocytes from leaving the skin. ${ }^{123}$ Consequently, the data suggest that CD69 induction in conjunction with downregulated S1P1 and the loss of CCR7 expression promotes retention of Trms in mucosal sites.

$\mathrm{CD} 103$ is also likely to have a significant role in retention and perhaps initial entry of Trms into various tissues (Figures $\mathbf{1}$ and 2). The only known ligand for CD103 is E-cadherin, ${ }^{127,128}$ which is expressed by epithelial cells throughout the body, as well as by a subset of DCs. ${ }^{129}$ Adoptive transfer studies with lymphocytes from CD103-deficient mice show that this integrin is not required for CD8 $\mathrm{T}$ cells to enter the intestinal mucosa, ${ }^{90,130}$ even though the mucosal tissues of CD103deficient mice contain fewer CD8 $\mathrm{T}$ cells than wild-type animals. ${ }^{59}$ In a model of graft-vs.-host disease, alloreactive CD8 $\mathrm{T}$ cells are gradually lost from the epithelium of the intestine when CD103 is absent. ${ }^{58}$ In addition, when CD103-deficient CD8 T cells respond to a systemic infection with Listeria, they are retained in the LP but not in the IEL compartment, ${ }^{90}$ despite the fact that some LP-resident CD8 T cells express CD103. In contrast, our previous results suggest that CD103 is not required for maintenance of at least some early memory cells in the epithelium (3 weeks postsystemic infection) ${ }^{130}$ but our recent studies corroborate the partial effect of CD103 deficiency on the loss of IEL (B.S. Sheridan and L. Lefrançois, personal communication), which we initially observed and may have misinterpreted. Additional studies are needed to determine whether CD103 has a role during early entry or retention of effector CD8 $\mathrm{T}$ cells in the intestinal epithelium vs. the longterm maintenance of memory CD8 $\mathrm{T}$ cells at this site. Similar to CD69 expression, the question of whether antigen is required for CD103 induction remains open and may be both tissue- and pathogen-dependent. Experiments with lymphocytic choriomeningitis virus-infected mice originally suggested that antigen was not required for maintenance of CD103 expression on CD8 $\mathrm{T}$ cells in the intestinal mucosa and other tissues, including the brain. ${ }^{90}$ However, following direct infection of the brain with VSV, CD103 expression is maintained on antigen-specific CD8 $\mathrm{T}$ cells only as long as the antigen is present. ${ }^{87}$ Interestingly, influenza virus-specific CD8 T cells in the epithelial layer of the human lung express CD103, whereas Epstein-Barr virus- and cytomegalovirus-specific CD8 T cells do not. ${ }^{131}$ As the latter infections are chronic, the presence of antigen in the lungs may be insufficient for CD103 induction in this case. Why antigen appears to be necessary to drive T cells to upregulate CD103 expression in some cases and not others remains to be elucidated. Recent studies show that most memory CD8 T cells in the dermis of the skin also express CD103, which appears important for their retention at that site. ${ }^{112}$ Some CD8 T cells in the human epidermal layer also express CD69 together with CD103. ${ }^{132}$ Thus, the expression of CD103 and CD69 has emerged as a hallmark of Trms and may be a critical component of protective immunity by promoting Trms' retention in multiple tissues, including the mucosae.
The initial induction of CD103 expression on T cells, including on Tregs, requires TGF $\beta,{ }^{51,58}$ which is produced by a variety of cells in the lung and intestines, including epithelial cells. ${ }^{58,91}$ CD103 + DCS can also produce TGF $\beta$ in the local LNs or other tissues, which may stimulate CD103 expression on activated CTLs in the lungs and other sites. ${ }^{58,91}$ TGF $\beta$ may also be made by the responding $\mathrm{T}$ cells themselves, ${ }^{133,134}$ in a latent form, which must be activated to exert function. ${ }^{135}$ Several proteases and integrins can perform this cleavage function, including the $\alpha \mathrm{V} \beta 8$ integrin that is expressed by CD103 + DCs as well as other cells. ${ }^{136,137}$ Large quantities of TGF $\beta$ can also be activated in the lungs during infection with some strains of influenza and other respiratory viruses by the virally encoded neuraminidase enzyme that is required to release new virus particles from the host cells. ${ }^{138,139}$ Consequently, the presence of TGF $\beta$ in mucosal tissues likely serves to promote retention of Trms in the appropriate locations via induction of CD103, thereby promoting protective immunity (Figures 1 and 2). There may also be a link between CD69 expression and TGF $\beta$ production. A recent report shows that $\mathrm{CD} 69$ promotes TGF $\beta$ production by intestinal CD4 $\mathrm{T}$ cells, but whether this is a direct effect requires further analysis. ${ }^{106}$ If CD69 activation does, in fact, regulate TGF $\beta$ production, then this pathway may represent a reinforcing loop geared toward maintaining CD103 induction. Additional studies are needed to determine the link between T-cell entry into mucosal sites and concomitant induction of CD69 and CD103. Thus, the current data suggest that protective Trms are maintained in the peripheral tissues through the concerted action of multiple molecules, such as CD69, CD103, S1P1, and CCR7.

\section{TRMS HAVE A ROLE IN PROTECTIVE IMMUNITY IN PERIPHERAL TISSUES}

Direct evidence that Trms have an essential role in protective immunity in peripheral tissues is largely lacking. So far, the strongest evidence comes from studies of skin infections, where virus-specific CD103 + CD8 T cells are sufficient to prevent a local vaccinia virus infection. ${ }^{89}$ In the intestinal mucosa, it is more difficult to pinpoint a precise role in protective immunity for Trms in the LP or the epithelium. This is largely due to the technical challenges of relegating functionality exclusively to either compartment. So far, there are no available methods for blocking migration of effector or memory cells only into the epithelium. Inhibition of CCR9 or $\alpha 4 \beta 7$ generally results in limiting migration to both LP and epithelium and can also affect homing to MLNs and PPs. ${ }^{130,140}$ Nevertheless, animal models have convincingly shown that blood-borne lymphocytes are not sufficient for optimum T-cell-mediated immunity against infections that are initiated in the lungs or intestines. These studies found that mucosal routes of inoculation induce more effective immunity than do systemic infections, even though both routes of inoculation induce large numbers of microbe-specific memory $\mathrm{T}$ cells in the circulation. ${ }^{141-147}$ No doubt immunity in the GI tract and skin and perhaps the lung is enhanced by antigen-presenting cells, which promote T-cell migration to localized tissues through the imprinting 
process. ${ }^{48,49,61,148-150}$ As always there are exceptions to the rule since mucosal or systemic vaccination is effective against mucosal challenge with mouse norovirus; however, CD4 and CD8 $\mathrm{T}$ cells, as well as antibody, are involved in the protection. ${ }^{151}$ In addition, a recent study found that the numbers of circulating influenza virus-specific CD4 T cells in human blood correlate more tightly with protective immunity than do the numbers of virus-specific CD8 T cells or antibody titers. ${ }^{152}$ In this study, the authors were careful to emphasize that virus-specific $\mathrm{T}$ cells at the site of infection were not examined, ${ }^{152}$ so the correlation between high numbers of CD4 $\mathrm{T}$ cells in the blood and the numbers of CD4 or CD8 T cells in the relevant tissue remains unknown. In animal models, transfer studies have shown that virus-specific CD4 T cells can augment antiviral immunity in the lungs of naive mice, without transferred CD8 T cells. ${ }^{21,64,104,105}$ As lung epithelial cells do not express major histocompatiblity complex class II molecules, the protection in this case may have been due to an accelerated response by the endogenous immune cells. ${ }^{153}$ CD4 $\mathrm{T}$ cells are also important in protection against herpes simplex virus infection of the vaginal mucosa. ${ }^{154,155}$ In any case, the available data provide reasonable support for the concept that both CD4 and CD8 Trms mediate local protective immunity. More detailed analyses of the potential synergy between T-cell subsets will help reveal the underpinnings of tissue-specific immunity. Focusing vaccine strategies on promoting production of these populations of $\mathrm{T}$ cells may enhance immunity.

\section{Summary}

Tissue-specific infections result in the generation of tissueresident populations of pathogen-specific memory CD4 and CD8 T cells. In most situations, these populations appear not be replenished to a great extent by circulating $\mathrm{T}$ cells nor do they enter the circulation themselves after the infectious agent is eliminated. As such, Trms represent a primarily self-renewing protective barrier against recurrent infections. Cells with these common attributes colonize the mucosa of the lung, intestine, and vagina, and are also found in nonmucosal sites, including the skin and brain, and may perhaps, depending on the pathogen and infection route, reside in any nonlymphoid tissue. Nonetheless, the barrier mucosal tissues and the skin are critical to providing defense against recurrent invasions and Trms would be expected to have a major protective role at these sites.

\section{ACKNOWLEDGEMENTS}

This work was supported by grants from the National Institutes of Health.

\section{DISCLOSURE}

The authors declared no conflict of interest.

c) 2013 Society for Mucosal Immunology

\section{REFERENCES}

1. Stiver, H.G. The threat and prospects for control of an influenza pandemic. Expert Rev. Vaccines 3, 35-42 (2004).

2. Monsalvo, A.C. et al. Severe pandemic $2009 \mathrm{H} 1 \mathrm{~N} 1$ influenza disease due to pathogenic immune complexes. Nat. Med. 17, 195-199 (2011).
3. Kolling, G., Wu, M. \& Guerrant, R.L. Enteric pathogens through life stages. Front. Cell Infect. Microbiol. 2, 114 (2012).

4. Neutra, M.R., Pringault, E. \& Kraehenbuhl, J.P. Antigen sampling across epithelial barriers and induction of mucosal immune responses [Review] [148 refs]. Annu. Rev. Immunol. 14, 275-300 (1996).

5. Neutra, M.R., Frey, A. \& Kraehenbuhl, J.P. Epithelial M cells: gateways for mucosal infection and immunization. Cell 86, 345-348 (1996).

6. Hansson, G.C. Role of mucus layers in gut infection and inflammation. Curr. Opin. Microbiol. 15, 57-62 (2012).

7. Lehrer, R.I. \& Lu, W. Alpha-Defensins in human innate immunity. Immunol. Rev. 245, 84-112 (2012).

8. Iwasaki, A. \& Medzhitov, R. Regulation of adaptive immunity by the innate immune system. Science 327, 291-295 (2010).

9. Kinnebrew, M.A. \& Pamer, E.G. Innate immune signaling in defense against intestinal microbes. Immunol. Rev. 245, 113-131 (2012).

10. Pott, J. \& Hornef, M. Innate immune signalling at the intestinal epithelium in homeostasis and disease. EMBO Rep. 13, 684-698 (2012).

11. Gallo, R.L. \& Hooper, L.V. Epithelial antimicrobial defence of the skin and intestine. Nat. Rev. Immunol. 12, 503-516 (2012).

12. Yoon, H., Legge, K.L., Sung, S.S. \& Braciale, T.J. Sequential activation of CD8 + Tcells in the draining lymph nodes in response to pulmonary virus infection. J. Immunol. 179, 391-399 (2007).

13. Milling, S., Yrlid, U., Cerovic, V. \& MacPherson, G. Subsets of migrating intestinal dendritic cells. Immunol. Rev. 234, 259-267 (2010).

14. Yoon, H., Kim, T.S. \& Braciale, T.J. The cell cycle time of CD8 + T cells responding in vivo is controlled by the type of antigenic stimulus. PLOS One 5, e154232010).

15. Sallusto, F., Lanzavecchia, A., Araki, K. \& Ahmed, R. From vaccines to memory and back. Immunity 33, 451-463 (2010).

16. Pepper, M. \& Jenkins, M.K. Origins of CD4(+) effector and central memory T cells. Nat. Immunol. 12, 467-471 (2011).

17. Rutishauser, R.L. \& Kaech, S.M. Generating diversity: transcriptional regulation of effector and memory CD8 T-cell differentiation. Immunol. Rev. 235, 219-233 (2010).

18. Woodland, D.L. \& Kohlmeier, J.E. Migration, maintenance and recall of memory T cells in peripheral tissues. Nat. Rev. Immunol. 9, 153-161 (2009).

19. lijima, N. et al. Dendritic cells and B cells maximize mucosal Th1 memory response to herpes simplex virus. J. Exp. Med. 205, 3041-3052 (2008).

20. Kumamoto, Y. \& Iwasaki, A. Unique features of antiviral immune system of the vaginal mucosa. Curr. Opin. Immunol. 24, 411-416 (2012).

21. Swain, S.L., McKinstry, K.K. \& Strutt, T.M. Expanding roles for $\mathrm{CD} 4(+) \mathrm{T}$ cells in immunity to viruses. Nat. Rev. Immunol. 12, 136-148 (2012).

22. Ariotti, S., Haanen, J.B. \& Schumacher, T.N. Behavior and function of tissue-resident memory T cells. Adv. Immunol. 114, 203-216 (2012).

23. Stephens, R., Randolph, D.A., Huang, G., Holtzman, M.J. \& Chaplin, D.D. Antigen-nonspecific recruitment of Th2 cells to the lung as a mechanism for viral infection-induced allergic asthma. J. Immunol. 169, 5458-5467 (2002).

24. Ely, K.H. et al. Nonspecific recruitment of memory CD8(+) T cells to the lung airways during respiratory virus infections. J. Immunol. 170, 1423-1429 (2003).

25. Masopust, D. et al. Activated primary and memory CD8 T cells migrate to nonlymphoid tissues regardless of site of activation or tissue of origin. J. Immunol. 172, 4875-4882 (2004).

26. Mowat, A.M. Anatomical basis of tolerance and immunity to intestinal antigens. Nat. Rev. Immunol. 3, 331-341 (2003).

27. Sheridan, B.S. \& Lefrancois, L. Regional and mucosal memory T cells. Nat. Immunol. 12, 485-491 (2011).

28. Lorenz, R.G. \& Newberry, R.D. Isolated lymphoid follicles can function as sites for induction of mucosal immune responses. Ann. NY Acad. Sci. 1029, 44-57 (2004).

29. Knoop, K.A., Butler, B.R., Kumar, N., Newberry, R.D. \& Williams, I.R. Distinct developmental requirements for isolated lymphoid follicle formation in the small and large intestine: RANKL is essential only in the small intestine. Am. J. Pathol. 179, 1861-1871 (2011).

30. Mowat, A.M., Millington, O.R. \& Chirdo, F.G. Anatomical and cellular basis of immunity and tolerance in the intestine. J. Pediatr. Gastroenterol. Nutr. 39 (Suppl 3)), S723-S724 (2004). 
31. Belz, G.T. et al. Distinct migrating and nonmigrating dendritic cell populations are involved in $\mathrm{MHC}$ class I-restricted antigen presentation after lung infection with virus. Proc. Natl. Acad. Sci. USA 101, 8670-8675 (2004).

32. Kim, T.S. \& Braciale, T.J. Respiratory dendritic cell subsets differ in their capacity to support the induction of virus-specific cytotoxic CD8 + Tcell responses. PLoS One 4, e4204 (2009).

33. Ballesteros-Tato, A., Leon, B., Lund, F.E. \& Randall, T.D. Temporal changes in dendritic cell subsets, cross-priming and costimulation via CD70 control CD8 $(+)$ T cell responses to influenza. Nat. Immunol. 11, 216-224 (2010).

34. de Heer, H.J., Hammad, H., Kool, M. \& Lambrecht, B.N. Dendritic cell subsets and immune regulation in the lung. Semin. Immunol. 17, 295-303 (2005).

35. von Garnier, C. et al. Anatomical location determines the distribution and function of dendritic cells and other APCs in the respiratory tract. J. Immunol. 175, 1609-1618 (2005).

36. Debertin, A.S. et al. Nasal-associated lymphoid tissue (NALT): frequency and localization in young children. Clin. Exp. Immunol. 134, 503-507 (2003).

37. Wiley, J.A., Tighe, M.P. \& Harmsen, A.G. Upper respiratory tract resistance to influenza infection is not prevented by the absence of either nasal-associated lymphoid tissue or cervical lymph nodes. J. Immunol. 175, 3186-3196 (2005).

38. Fernandez, S., Cisney, E.D., Hall, S.I. \& Ulrich, R.G. Nasal immunity to staphylococcal toxic shock is controlled by the nasopharynx-associated lymphoid tissue. Clin. Vaccine Immunol. 18, 667-675 (2011).

39. Moyron-Quiroz, J.E. et al. Role of inducible bronchus associated lymphoid tissue (iBALT) in respiratory immunity. Nat. Med. 10, 927-934 (2004).

40. Kim, D.Y. et al. The airway antigen sampling system: respiratory M cells as an alternative gateway for inhaled antigens. J. Immunol. 186, 4253-4262 (2011).

41. Foo, S.Y. \& Phipps, S. Regulation of inducible BALT formation and contribution to immunity and pathology. Mucosal Immunol. 3, 537-544 (2010).

42. Swiatczak, B. \& Rescigno, M. How the interplay between antigen presenting cells and microbiota tunes host immune responses in the gut. Semin. Immunol. 24, 43-49 (2012).

43. Cerovic, V. et al. Intestinal CD103( - ) dendritic cells migrate in lymph and prime effector T cells. Mucosal. Immunol. 2012).

44. GeurtsvanKessel, C.H. et al. Clearance of influenza virus from the lung depends on migratory langerin $+\mathrm{CD} 11 \mathrm{~b}-$ but not plasmacytoid dendritic cells. J. Exp. Med. 205, 1621-1634 (2008).

45. Scott, C.L., Aumeunier, A.M. \& Mowat, A.M. Intestinal CD103 + dendritic cells: master regulators of tolerance?. Trends Immunol. 32, 412-419 (2011).

46. Cerovic, V. et al. New insights into the roles of dendritic cells in intestinal immunity and tolerance. Int. Rev. Cell Mol. Biol. 272, 33-105 (2009).

47. Stagg, A.J., Kamm, M.A. \& Knight, S.C. Intestinal dendritic cells increase Tcell expression of alpha4beta7 integrin. Eur. J. Immunol. 32, 1445-1454 (2002).

48. Mora, J.R. et al. Selective imprinting of gut-homing T cells by Peyer's patch dendritic cells. Nature 424, 88-93 (2003).

49. Johansson-Lindbom, B. et al. Functional specialization of gut CD103+ dendritic cells in the regulation of tissue-selective T cell homing. J. Exp. Med. 202, 1063-1073 (2005).

50. Iwata, M. et al. Retinoic acid imprints gut-homing specificity on T cells. Immunity 21, 527-538 (2004).

51. Coombes, J.L. et al. A functionally specialized population of mucosal CD103 + DCs induces Foxp3 + regulatory T cells via a TGF-beta and retinoic acid-dependent mechanism. J. Exp. Med. 204, 1757-1764 (2007).

52. Svensson, M. et al. Retinoic acid receptor signaling levels and antigen dose regulate gut homing receptor expression on CD8 + Tcells. Mucosal Immunol. 1, 38-48 (2008).

53. Kang, S.G., Park, J., Cho, J.Y., Ulrich, B. \& Kim, C.H. Complementary roles of retinoic acid and TGF-beta1 in coordinated expression of mucosal integrins by T cells. Mucosal. Immunol. 4, 66-82 (2011).

54. Iwata, M. \& Yokota, A. Retinoic acid production by intestinal dendritic cells. Vitam. Horm. 86, 127-152 (2011).
55. Kunkel, E.J. et al. Lymphocyte CC chemokine receptor 9 and epithelial thymus-expressed chemokine (TECK) expression distinguish the small intestinal immune compartment: epithelial expression of tissue-specific chemokines as an organizing principle in regional immunity. J. Exp. Med. 192, 761-768 (2000).

56. Gorfu, G., Rivera-Nieves, J. \& Ley, K. Role of beta7 integrins in intestinal lymphocyte homing and retention. Curr. Mol. Med. 9, 836-850 (2009).

57. Ericsson, A., Svensson, M., Arya, A. \& Agace, W.W. CCL25/CCR9 promotes the induction and function of CD103 on intestinal intraepithelial lymphocytes. Eur. J. Immunol. 34, 2720-2729 (2004).

58. El Asady, R. et al. TGF-\{beta\}-dependent CD103 expression by CD8(+) $T$ cells promotes selective destruction of the host intestinal epithelium during graft-versus-host disease. J. Exp. Med 201, 1647-1657 (2005).

59. Schon, M.P. et al. Mucosal T lymphocyte numbers are selectively reduced in integrin alpha E (CD103)-deficient mice. J. Immunol. 162, 6641-6649 (1999).

60. Kim, S.K., Schluns, K.S. \& Lefrancois, L. Induction and visualization of mucosal memory CD8 $T$ cells following systemic virus infection. J. Immunol. 163, 4125-4132 (1999).

61. Campbell, D.J. \& Butcher, E.C. Rapid acquisition of tissue-specific homing phenotypes by CD4 $(+)$ Tcells activated in cutaneous or mucosal lymphoid tissues. J. Exp. Med. 195, 135-141 (2002).

62. Kunisawa, J. et al. Sphingosine 1-phosphate dependence in the regulation of lymphocyte trafficking to the gut epithelium. J. Exp. Med. 204, 2335-2348 (2007).

63. GeurtsvanKessel, C.H. \& Lambrecht, B.N. Division of labor between dendritic cell subsets of the lung. Mucosal Immunol. 1, 442-450 (2008).

64. Teijaro, J.R. et al. Cutting edge: tissue-retentive lung memory CD4 T cells mediate optimal protection to respiratory virus infection. J. Immunol. 187, 5510-5514 (2011).

65. Jungi, T.W. \& Jungi, R. Immunological memory to Listeria monocytogenes in rodents. IV. Studies on origin and fate of tissue-positioned T memory cells. Immunology 44, 789-798 (1981).

66. Donskoy, E. \& Goldschneider, I. Thymocytopoiesis is maintained by blood-borne precursors throughout postnatal life. A study in parabiotic mice. J. Immunol. 148, 1604-1612 (1992).

67. Ansel, K.M., Harris, R.B. \& Cyster, J.G. CXCL13 is required for B1 cell homing, natural antibody production, and body cavity immunity. Immunity 16, 67-76 (2002).

68. Klonowski, K.D. et al. Dynamics of blood-borne CD8 memory T cell migration in vivo. Immunity 20, 551-562 (2004).

69. Poussier, P., Edouard, P., Lee, C., Binnie, M. \& Julius, M. Thymusindependent development and negative selection of T cells expressing T cell receptor $\alpha / \beta$ in the intestinal epithelium: evidence for distinct circulation patterns of gut- and thymus-derived $T$ lymphocytes. J. Exp. Med. 176, 187-199 (1992).

70. Masopust, D., Vezys, V., Wherry, E.J., Barber, D.L. \& Ahmed, R. Cutting edge: gut microenvironment promotes differentiation of a unique memory CD8 T cell population. J. Immunol. 176, 2079-2083 (2006).

71. Masopust, D. et al. Dynamic T cell migration program provides resident memory within intestinal epithelium. J. Exp. Med. 207, 553-564 (2010).

72. Moyron-Quiroz, J.E. et al. Persistence and responsiveness of immunologic memory in the absence of secondary lymphoid organs. Immunity 25 , 643-654 (2006).

73. Zammit, D.J., Turner, D.L., Klonowski, K.D., Lefrancois, L. \& Cauley, L.S. Residual antigen presentation after influenza virus infection affects CD8 T cell activation and migration. Immunity 24, 439-449 (2006).

74. Anderson, K.G. et al. Cutting edge: intravascular staining redefines lung CD8 T cell responses. J. Immunol. 189, 2702-2706 (2012).

75. Jelly-Gibbs, D.M. et al. Unexpected prolonged presentation of influenza antigens promotes CD4 Tcell memory generation. J. Exp. Med. 202, 697706 (2005).

76. Kim, T.S., Hufford, M.M., Sun, J., Fu, Y.X. \& Braciale, T.J. Antigen persistence and the control of local $T$ cell memory by migrant respiratory dendritic cells after acute virus infection. J. Exp. Med. 207, 1161-1172 (2010).

77. Lefrancois, L., Altman, J.D., Williams, K. \& Olson, S. Soluble antigen and CD40 triggering are sufficient to induce primary and memory cytotoxic T cells. J. Immunol. 164, 725-732 (2000).

78. Maxwell, J.R., Rossi, R.J., McSorley, S.J. \& Vella, A.T. T cell clonal conditioning: a phase occurring early after antigen presentation but 
before clonal expansion is impacted by Toll-like receptor stimulation. J. Immunol. 172, 248-259 (2004).

79. Jabbari, A., Legge, K.L. \& Harty, J.T. T cell conditioning explains early disappearance of the memory CD8 T cell response to infection. J. Immunol. 177, 3012-3018 (2006).

80. Turner, D.L., Cauley, L.S., Khanna, K.M. \& Lefrancois, L. Persistent antigen presentation after acute vesicular stomatitis virus infection. J. Virol. 81, 2039-2046 (2007).

81. Simon, I.D., Publicover, J. \& Rose, J.K. Replication and propagation of attenuated vesicular stomatitis virus vectors in vivo: vector spread correlates with induction of immune responses and persistence of genomic RNA. J. Virol. 81, 2078-2082 (2007).

82. Simon, I.D., van, R.N. \& Rose, J.K. Vesicular stomatitis virus genomic RNA persists in vivo in the absence of viral replication. J. Virol. 84, 32803286 (2010).

83. Hadley, G. Role of integrin CD103 in promoting destruction of renal allografts by CD8 T cells. Am. J. Transplant. 4, 1026-1032 (2004).

84. Feng, Y. et al. CD103 expression is required for destruction of pancreatic islet allografts by CD8(+) T cells. J. Exp. Med. 196, 877-886 (2002).

85. Lee, H.K. et al. Differential roles of migratory and resident DCs in T cell priming after mucosal or skin HSV-1 infection. J. Exp. Med. 206, 359-370 (2009).

86. Gebhardt, T. et al. Memory T cells in nonlymphoid tissue that provide enhanced local immunity during infection with herpes simplex virus. Nat. Immunol. 10, 524-530 (2009).

87. Wakim, L.M., Woodward-Davis, A. \& Bevan, M.J. Memory T cells persisting within the brain after local infection show functional adaptations to their tissue of residence. Proc. Natl. Acad. Sci. USA 107, 17872-17879 (2010).

88. Purwar, R. et al. Resident memory T cells (T(RM)) are abundant in human lung: diversity, function, and antigen specificity. PLoS One 6, e16245 (2011).

89. Jiang, $X$. et al. Skin infection generates non-migratory memory CD8 + TRM cells providing global skin immunity. Nature 483, 227-231 (2012).

90. Casey, K.A. et al. Antigen-independent differentiation and maintenance of effector-like resident memory T cells in tissues. J. Immunol. 188, 48664875 (2012).

91. Lee, Y.T. et al. Environmental and antigen-receptor derived signals support sustained surveillance of the lungs by pathogen-specific CTL. J. Virol. 85, 4085-4094 (2011).

92. Goodman, T. \& Lefrancois, L. Intraepithelial lymphocytes. Anatomical site, not T cell receptor form, dictates phenotype and function. J. Exp. Med. 170, 1569-1581 (1989).

93. Lefrançois, L. Phenotypic complexity of intraepithelial lymphocytes of the small intestine. J. Immunol. 147, 1746-1751 (1991).

94. Badiner, G., Goodman, T.G. \& Lefrançois, L. Selection of intestinal intraepithelial lymphocyte T-cell receptors: evidence for a dynamic tissuespecific process. Int. Immunol. 5, 223-226 (1993).

95. Huleatt, J.W., Pilip, I., Kerksiek, K. \& Pamer, E.G. Intestinal and splenic T cell responses to enteric Listeria monocytogenes infection: distinct repertoires of responding CD8 T lymphocytes. J. Immunol. 166, 40654073 (2001).

96. Kim, S.K., Reed, D.S., Heath, W.R., Carbone, F. \& Lefrancois, L. Activation and migration of CD8 T cells in the intestinal mucosa. J. Immunol. 159, 4295-4306 (1997).

97. Cauley, L.S. et al. Cutting edge: virus-specific CD4(+) memory T cells in nonlymphoid tissues express a highly activated phenotype. J. Immunol. 169, 6655-6658 (2002).

98. Hogan, R.J. et al. Activated antigen-specific CD8 + T cells persist in the lungs following recovery from respiratory virus infections. J. Immunol. 166, 1813-1822 (2001).

99. Ostler, T., Hussell, T., Surh, C.D., Openshaw, P. \& Ehl, S. Long-term persistence and reactivation of $T$ cell memory in the lung of mice infected with respiratory syncytial virus. Eur. J. Immunol. 31, 2574-2582 (2001).

100. Marshall, D.R. et al. Measuring the diaspora for virus-specific CD8 + T cells. Proc. Natl. Acad. Sci. USA 98, 6313-6318 (2001).

101. Ely, K.H., Roberts, A.D. \& Woodland, D.L. Cutting edge: effector memory CD8 + T cells in the lung airways retain the potential to mediate recall responses. J. Immunol. 171, 3338-3342 (2003).
102. Ely, K.H., Cookenham, T., Roberts, A.D. \& Woodland, D.L. Memory Tcell populations in the lung airways are maintained by continual recruitment. J. Immunol. 176, 537-543 (2006).

103. Homann, D., Teyton, L. \& Oldstone, M.B. Differential regulation of antiviral T-cell immunity results in stable CD8 + but declining CD4 + T-cell memory. Nat. Med. 7, 913-919 (2001).

104. Hogan, R.J. et al. Protection from respiratory virus infections can be mediated by antigen-specific CD4(+) T cells that persist in the lungs. J. Exp. Med. 193, 981-986 (2001).

105. Marshall, N.B. \& Swain, S.L. Cytotoxic CD4 T cells in antiviral immunity. J. Biomed. Biotechnol. 2011, 9546022011).

106. Radulovic, K. et al. CD69 regulates type I IFN-induced tolerogenic signals to mucosal CD4 $\mathrm{T}$ cells that attenuate their colitogenic potential. J. Immunol. 188, 2001-2013 (2012).

107. Sancho, D., Gomez, M. \& Sanchez-Madrid, F. CD69 is an immunoregulatory molecule induced following activation. Trends Immunol. 26, 136-140 (2005).

108. Craston, R. et al. Temporal dynamics of CD69 expression on lymphoid cells. J. Immunol. Methods 209, 37-45 (1997).

109. Deblandre, G.A., Leo, O., Huez, G.A. \& Wathelet, M.G. CD69 is expressed on Daudi cells in response to interferon-alpha. Cytokine 4, 36-43 (1992).

110. Wang, H.C., Zhou, Q., Dragoo, J. \& Klein, J.R. Most murine CD8+ intestinal intraepithelial lymphocytes are partially but not fully activated T cells. J. Immunol. 169, 4717-4722 (2002).

111. Sheridan, B.S. \& Lefrancois, L. Intraepithelial lymphocytes: to serve and protect. Curr. Gastroenterol. Rep. 12, 513-521 (2010).

112. Gebhardt, T. et al. Different patterns of peripheral migration by memory CD4 + and CD8 + T cells. Nature 477, 216-219 (2011).

113. Khanna, K.M., Bonneau, R.H., Kinchington, P.R. \& Hendricks, R.L. Herpes simplex virus-specific memory CD8 $+\mathrm{T}$ cells are selectively activated and retained in latently infected sensory ganglia. Immunity $\mathbf{1 8}$, 593-603 (2003)

114. St. Leger, A.J. \& Hendricks, R.L. CD8(+) T cells patrol HSV-1-infected trigeminal ganglia and prevent viral reactivation. J. Neurovirol. 17, 528534 (2011).

115. Kovalova, A. et al. Synthetic N-acetyl-D-glucosamine based fully branched tetrasaccharide, a mimetic of the endogenous ligand for CD69, activates CD69 + killer lymphocytes upon dimerization via a hydrophilic flexible linker. J. Med. Chem. 53, 4050-4065 (2010).

116. Nakayama, T. et al. The generation of mature, single-positive thymocytes in vivo is dysregulated by CD69 blockade or overexpression. J. Immunol. 168, 87-94 (2002).

117. Feng, C. et al. A potential role for CD69 in thymocyte emigration. Int. Immunol. 14, 535-544 (2002).

118. Shiow, L.R. et al. CD69 acts downstream of interferon-alpha/beta to inhibit S1P1 and lymphocyte egress from lymphoid organs. Nature 440 , 540-544 (2006).

119. Cyster, J.G. \& Schwab, S.R. Sphingosine-1-phosphate and lymphocyte egress from lymphoid organs. Annu. Rev. Immunol. 30, 69-94 (2012).

120. Bankovich, A.J., Shiow, L.R. \& Cyster, J.G. CD69 suppresses sphingosine 1-phosophate receptor-1 (S1P1) function through interaction with membrane helix 4. J. Biol. Chem. 285, 22328-22337 (2010).

121. Matloubian, M. et al. Lymphocyte egress from thymus and peripheral lymphoid organs is dependent on S1P receptor 1. Nature 427, 355-360 (2004).

122. Bromley, S.K., Thomas, S.Y. \& Luster, A.D. Chemokine receptor CCR7 guides Tcell exit from peripheral tissues and entry into afferent lymphatics. Nat. Immunol. 6, 895-901 (2005).

123. Debes, G.F. et al. Chemokine receptor CCR7 required for T lymphocyte exit from peripheral tissues. Nat. Immunol. 6, 889-894 (2005).

124. Hopken, U.E., Winter, S., Achtman, A.H., Kruger, K. \& Lipp, M. CCR7 regulates lymphocyte egress and recirculation through body cavities. J. Leukoc. Biol. 87, 671-682 (2010).

125. Martin-Fontecha, A. et al. Regulation of dendritic cell migration to the draining lymph node: impact on T lymphocyte traffic and priming. J. Exp. Med. 198, 615-621 (2003).

126. Jennrich, S., Lee, M.H., Lynn, R.C., Dewberry, K. \& Debes, G.F. Tissue exit: a novel control point in the accumulation of antigen-specific CD8 T cells in the influenza a virus-infected lung. J. Virol. 86, 3436-3445 (2012). 
127. Roberts, K. \& Kilshaw, P.J. The mucosal T cell integrin alpha M290 beta 7 recognizes a ligand on mucosal epithelial cell lines. Eur. J. Immunol 23 1630-1635 (1993).

128. Cepek, K.L. et al. Adhesion between epithelial cells and T lymphocytes mediated by E-cadherin and the alpha E beta 7 integrin. Nature 372, 190193 (1994).

129. Siddiqui, K.R., Laffont, S. \& Powrie, F. E-cadherin marks a subset of inflammatory dendritic cells that promote Tcell-mediated colitis. Immunity 32, 557-567 (2010).

130. Lefrancois, L. et al. The role of beta7 integrins in CD8 T cell trafficking during an antiviral immune response. J. Exp. Med. 189, 1631-1638 (1999).

131. Piet, B. et al. CD8 + T cells with an intraepithelial phenotype upregulate cytotoxic function upon influenza infection in human lung. J. Clin. Invest. 121, 2254-2263 (2011).

132. Mizukawa, Y. et al. Direct evidence for interferon-gamma production by effector-memory-type intraepidermal T cells residing at an effector site of immunopathology in fixed drug eruption. Am. J. Pathol. 161, 1337-1347 (2002).

133. Li, M.O., Wan, Y.Y. \& Flavell, R.A. T cell-produced transforming growth factor-beta1 controls T cell tolerance and regulates Th1- and Th17-cell differentiation. Immunity 26, 579-591 (2007).

134. Gutcher, I. et al. Autocrine transforming growth factor-beta1 promotes in vivo Th17 cell differentiation. Immunity 34, 396-408 (2011).

135. Jenkins, $\mathrm{G}$. The role of proteases in transforming growth factor-beta activation. Int. J. Biochem. Cell Biol. 40, 1068-1078 (2008).

136. Fjellbirkeland, L. et al. Integrin alphavbeta8-mediated activation of transforming growth factor-beta inhibits human airway epithelial proliferation in intact bronchial tissue. Am. J. Pathol. 163, 533-542 (2003).

137. Paidassi, H. et al. Preferential expression of integrin alphavbeta8 promotes generation of regulatory T cells by mouse CD103 + dendritic cells. Gastroenterology 141, 1813-1820 (2011).

138. Schultz-Cherry, S. \& Hinshaw, V.S. Influenza virus neuraminidase activates latent transforming growth factor beta. J. Virol. 70, 86248629 (1996).

139. Carlson, C.M. et al. Transforming growth factor-beta: activation by neuraminidase and role in highly pathogenic H5N1 influenza pathogenesis. PLoS Pathogen. 6, e1001136 (2010).

140. Stenstad, H., Svensson, M., Cucak, H., Kotarsky, K. \& Agace, W.W. Differential homing mechanisms regulate regionalized effector CD8alphabeta $+\mathrm{T}$ cell accumulation within the small intestine. Proc. Natl. Acad. Sci. USA 104, 10122-10127 (2007).

141. Belyakov, I.M. et al. The importance of local mucosal HIV-specific CD8(+) cytotoxic T lymphocytes for resistance to mucosal viral transmission in mice and enhancement of resistance by local administration of IL-12. J. Clin. Invest. 102, 2072-2081 (1998).

142. McCluskie, M.J. et al. Route and method of delivery of DNA vaccine influence immune responses in mice and non-human primates. Mol. Med. 5, 287-300 (1999).

143. Belyakov, I.M. \& Ahlers, J.D. What role does the route of immunization play in the generation of protective immunity against mucosal pathogens?. J. Immunol. 183, 6883-6892 (2009).

144. Estcourt, M.J., Letourneau, S., McMichael, A.J. \& Hanke, T. Vaccine route, dose and type of delivery vector determine patterns of primary CD8 + T cell responses. Eur. J. Immunol. 35, 2532-2540 (2005).

145. Jiang, J.Q., He, X.S., Feng, N. \& Greenberg, H.B. Qualitative and quantitative characteristics of rotavirus-specific CD8 $T$ cells vary depending on the route of infection. J. Virol. 82, 6812-6819 (2008).

146. Takamura, S. et al. The route of priming influences the ability of respiratory virus-specific memory CD8 + Tcells to be activated by residual antigen. J. Exp. Med. 207, 1153-1160 (2010).

147. Suda, T. et al. The route of immunization with adenoviral vaccine influences the recruitment of cytotoxic $T$ lymphocytes in the lung that provide potent protection from influenza A virus. Antiviral Res. 91, 252258 (2011).

148. Reiss, Y., Proudfoot, A.E., Power, C.A., Campbell, J.J. \& Butcher, E.C. CC chemokine receptor (CCR) 4 and the CCR10 ligand cutaneous T cellattracting chemokine (CTACK) in lymphocyte trafficking to inflamed skin. J. Exp. Med. 194, 1541-1547 (2001).

149. Kunkel, E.J., Campbell, D.J. \& Butcher, E.C. Chemokines in lymphocyte trafficking and intestinal immunity. Microcirculation 10, 313-323 (2003).

150. Johansson-Lindbom, B. et al. Selective generation of gut tropic T cells in gut-associated lymphoid tissue (GALT): requirement for GALT dendritic cells and adjuvant. J. Exp. Med. 198, 963-969 (2003).

151. Chachu, K.A., LoBue, A.D., Strong, D.W., Baric, R.S. \& Virgin, H.W. Immune mechanisms responsible for vaccination against and clearance of mucosal and lymphatic norovirus infection. PLoS Pathogen. 4, e1000236 (2008).

152. Wilkinson, T.M. et al. Preexisting influenza-specific CD4(+) T cells correlate with disease protection against influenza challenge in humans. Nat. Med. 18, 274-280 (2012).

153. Nakanishi, Y., Lu, B., Gerard, C. \& Iwasaki, A. CD8(+) T lymphocyte mobilization to virus-infected tissue requires CD4(+) T-cell help. Nature 462, 510-513 (2009).

154. Zhao, X. et al. Vaginal submucosal dendritic cells, but not Langerhans cells, induce protective Th1 responses to herpes simplex virus-2. J. Exp. Med. 197, 153-162 (2003).

155. Iwasaki, A. Antiviral immune responses in the genital tract: clues for vaccines. Nat. Rev. Immunol. 10, 699-711 (2010). 